\title{
Hip Abductor Tendinopathy: Work-Up and Open Versus Arthroscopic Repair of Hip Abductor
}

\author{
*Nicholas D Casscells, Joshua A Kotler and William F Postma \\ Georgetown University Hospital, USA
}

Submission: September 02, 2016; Published: September 19, 2016

*Corresponding author: Nicholas D Casscells, Georgetown University Hospital, 3800 Reservoir Rd NW, Washington, DC 20007, USA.

\begin{abstract}
The abductors of the hip notably the gluteus medius and gluteus minimus are paramount to hip stability and function, so much so they have been aptly termed the rotator cuff of the hip [1,2]. Tendinopathy of these structures is classified among a group of other diseases that also present as lateral sided hip pain called greater trochanteric pain syndrome (GTPS); this also encompasses snapping hip and trochanteric bursitis. Historically, patients with lateral sided hip pain have been presumed to have trochanteric bursitis and treated with anti inflammatory medications, physical therapy and corticosteroid injections [3]. Failure of conservative therapy, in many cases, can be attributable to tears in the abductors sometimes necessitating surgical intervention. Both arthroscopic and open repairs of the abductors have been shown to be effective treatments if conservative measures fail [1-4].
\end{abstract}

\section{Introduction}

Hip abductor tendinopathy encompasses a constellation of etiologies all of which present with the chief complaint of lateral hip pain. It is four times more common in women and is usually seen between the fourth and sixth decades of life $[5,6]$. Patients with osteoarthritis, iliotibial band tenderness, low back pain, and obesity are at an increased risk $[7,8]$. Hip abductor tendinopathy falls under a broader classification of greater trochanteric pain syndrome (GTPS). Etiologies of GTPS include gluteus medius and minimus tendinopathy, external coxa saltans (hip snapping), and greater trochanteric bursitis $[9,10]$. Domb et al. \& Williams et al. $[7,11]$ relate the incidence of GTPS is approximately 1.8 per 1,000 people and classically presents as pain on the lateral aspect of the hip that can radiate to the thigh and posteriorly. Chronic, progressive pain is more common than acute-onset pain from an identifiable traumatic event $[12,13]$.

Historically, the etiology of GTPS has been mistaken for trochanteric bursitis. It was not until several decades after Stegemann et al. \& Leonard et al. [7,14] described the symptoms of what is now known as GTPS in the vicinity of the trochanter major as "trochanteric syndrome" in the Journal of the American Medical Association. Since Stegemann and Leonard, advancements in clinical imaging techniques such as magnetic resonance imaging (MRI) and ultrasound have permitted more definitive diagnosis of lateral hip pain as GTPS and not a true trochanteric bursitis. As recently as 2008, Silva et al. [15] in the Journal of Clinical Rheumatology maintains that no histologic study of trochanteric bursitis has been reported in the literature.
GTPS includes several potential causes, one of which is hip abductor tendinopathy, insufficiency, and tears.

\section{Anatomy}

The four hip abductor muscles accomplish hip abduction: gluteus maximus, gluteus medius, gluteus minimus, and tensor fasciae latae. The superior gluteal nerve innervates all but the gluteus maximus, which is innervated by the inferior gluteal nerve. The gluteus medius and gluteus minimus are the most frequently injured and thus the focus of hip abductor insufficiency. They are so commonly the culprit that the two have been termed the "rotator cuff tears of the hip" [1,2]. The gluteus medius is comprised of an anterior, middle and posterior portion. The anterior and middle portions are involved in initiating hip abduction while the anterior segment alone is the primary pelvic rotator [16]. The posterior portion of the gluteus medius stabilizes the hip from the heel strike to full stance during normal gait [16]. The gluteus minimus also stabilizes the hip joint during the gait cycle - specifically during the mid and late stages of gait [16].

\section{Differential diagnosis}

Abductor insufficiency tends to present as one of three clinical scenarios. First, older patients may present with lateral hip pain and abduction weakness without a history of arthritis $[16,17]$. In these patients, chronic, degenerative abductor tears should be considered - particularly in those who have failed conservative therapy $[16,17]$. A minority of patients may recall 


\section{Orthopedics and Rheumatology Open Access Journal}

a traumatic event and in such cases history of such an event is helpful in categorizing the tear in this first group [16,17]. These degenerative and symptomatic tears will be the focus of our discussion. Abductor tears in the second group of patients are found incidentally at the time of a hip arthroplasty and are asymptomatic $[16,18]$. The last group presents with symptoms of abductor tendon insufficiency following hip arthroplasty $[16,19,20]$. As the hip abductor tendons are partially or fully released to facilitate adequate exposure and then repaired prior to closure, the failure of repair can be responsible for abductor insufficiency $[16,19,20]$. In addition to the expected damage and subsequent repair of the hip abductors requisite of a THA, Hardinge et al. $[16,19,20]$ underscores that the anterolateral approach used in the procedure has been implicated in injury to the superior gluteal nerve - responsible for innervation of the gluteus medius and gluteus minimus - and its vessels.

Other etiologies of GTPS should be considered in the differential diagnosis. If snapping of the iliotibial band can be elicited on physical exam, a diagnosis of external coxa saltans should be considered. The maneuver is executed by moving the leg from a position of flexion, abduction, and external rotation to a position of extension, abduction, and internal rotation [9]. Outside of GTPS, pain that begins in the lower back and radiates to the lateral hip and thigh can be radicular and indicate lumbar spine pathology such as stenosis or spondylosis [16]. Other extra-articular diagnoses on the differential could include occult proximal femur fracture, injury to the superior gluteal nerve, or meralgia paresthetica. Decreased passive range of motion could indicate osteoarthritis of the hip, particularly if pain is also present in the groin. Other intra-articular pathologies on the differential include femoroacetabular impingement, labral tears, capsular laxity, chondral damage, and ligamentum teres rupture [9].

\section{History and physical}

A relevant history in the evaluation of a patient with suspected abductor tendinopathy will include a history of present illness detailing time-course of pain, characterization of pain, and interventions the patient has tried which either relieve or worsen pain. One should inquire of the use of steroid injections for presumed "trochanteric bursitis." In a history indicative of neuropathy, myelitis should be suspected. An experience of snapping, catching, clicking, locking, and popping should also be solicited [9]. A patient's past surgical treatment of the hip is important as certain surgeries can cause abductor insufficiency secondary to the approach as mentioned previously. As well, any history of attempted treatment - nonsurgical included - should be documented as this will impact future treatment options.

Patients presenting with GTPS secondary to hip abductor insufficiency endorse pain with weight bearing and difficulty ascending and descending stairs [5,12]. Narrowing the diagnosis, the examiner will notice point tenderness in the posterolateral area of the greater trochanter as well as pain with resisted hip abduction $[5,12,13,17]$. The examiner can also expect gait disturbances such as antalgic gait with or without Trendelenburg gait [5,7,12,13]. A 2001 study by Bird et al. [5] published in Arthritis and Rheumatology found Tendelenburg's sign to be the most sensitive and specific physical exam finding to gluteus medius tears. Physical exam results were assessed against MRI as a surrogate gold standard in 24 patients recruited for clinical features suggestive of GTPS [5]. Trendelenburg's sign was found to be $72.7 \%$ sensitive and $76.9 \%$ specific in detecting gluteus medius tears [5]. The Trendelenburg sign is elicited by having the patient lift the nonaffected foot; the pelvis subsequently drops or tilts to the nonaffected hip denoting insufficiency of the abductors to keep the pelvis level.

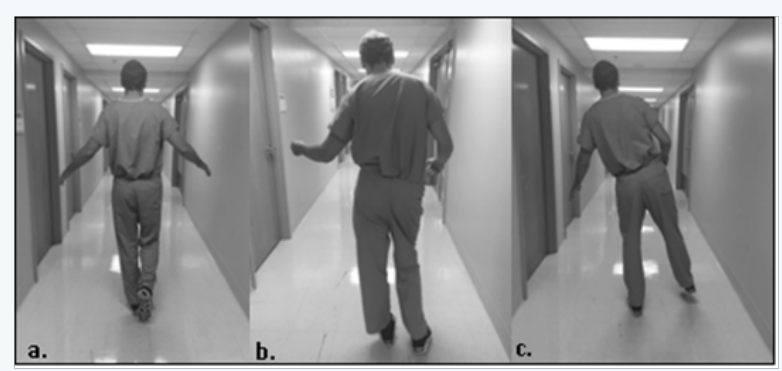

Figure 1: (a) Demonstrates a negative trendelenburg test with a normal level pelvis.

(b) Demonstrates a positive trendelenburg test on the left side in which insufficiency of the abductors on the left side of the hip cause the right side of the pelvis to drop.

(c) Shows a left sided trendelenburg gait which compensates for the abductor weakness on the left by leaning to left side during midstance so that the right leg can clear the ground.

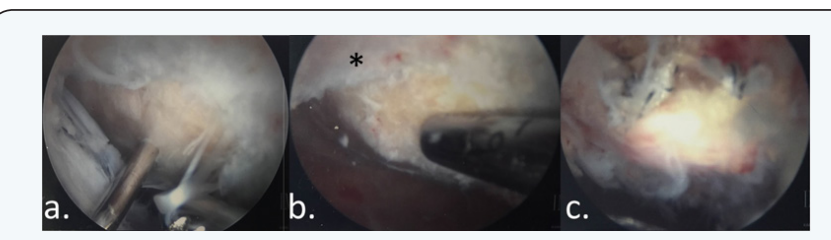

Figure 2: (a) Demonstrates a partial gluteus medial tear.

(b) Shows the preparation of the lateral facet of the greater trochanter with the gluteus medius marked by the $\left({ }^{*}\right)$.

(c) Illustrates the repaired gluteus medius utilizing two suture anchors.

The Trendelenburg gait is a compensatory mechanism in which the patient leans to the affected side when elevating the opposite leg in an attempt to keep the pelvis level during ambulation to compensate for abductor insufficiency as shown in (Figures 1 \& 2) [9,21]. This physical finding was evaluated alongside pain findings with resisted hip abduction and resisted hip internal rotation. Utility of Trendelenburg's sign eclipsed that of the other two maneuvers where pain experienced in resisted hip abduction demonstrated $72.7 \%$ sensitivity though only 46.2 $\%$ specificity and pain on resisted hip internal rotation sported only $54.5 \%$ sensitivity and $69.2 \%$ specificity [5]. Diagnosis can also be confirmed with peritrochanteric injections where the patient's pain is relieved while weakness of hip abduction persists [12,22]. 


\section{Imaging}

Radiographs are typically unremarkable in patients with abductor tears, but are often the first step in evaluating for other pathologies on the differential, such as osteoarthritis of the hip or fracture [16]. MRI has proven to be a highly specific diagnostic tool in the work-up of GTPS. In a 2004 retrospective study published in the American Journal of Roentgenology, 74 hips - 15 surgically confirmed abductor tendon tears and 59 confirmed intact tendons - were evaluated to determine the accuracy of MRI in diagnosing abductor tendon insufficiency [6]. Cvitanic et al. [6] showed that MRI findings with an area of T2 hyperintensity superior to the greater trochanter was $73 \%$ sensitive and 95 $\%$ specific to tears of the gluteus medius and gluteus minimus tendons. MRI can also be used in a patient suspected of GTPS to evaluate tendinosis, partial-thickness tear, or a full-thickness tear with loss of continuity $[6,12]$.

A thickened tendon and increased signal intensity on T2 weighted images were characteristic of tendinosis. Partial thickness tears were indicated by focal discontinuity of gluteus medius fibers, and complete tears were defined as those involving retraction of the tendon [9]. Abductor tears may also show hypertrophied tensor fasciae latae compared with contralateral side as a result of compensation for decreased abductor strength [9,23]. Redmond et al. [9] explains ultrasound has been shown to be an effective tool for GTPS and diagnosing abductor tendinopathy with a sensitivity ranging from $79 \%$ to $100 \%$ with a positive predictive value ranging from $95 \%$ to $100 \%$. Ultrasound as a dynamic evaluation modality can visualize external snapping of the hip. In a study of 877 patients with GTPS, Long et al. [24] used ultrasound to identify gluteal tendinosis in $50 \%$ of patients, a thickened iliotibial band in 28.5 $\%$ of patients, a gluteal tear in $0.5 \%$ of patients, and trochanteric bursitis in $20 \%$ of patients. Incidentally, Long et. al concluded that only a minority of patients (20.2\%) were determined to have trochanteric bursitis [24].

\section{Treatment}

In patients with GTPS, including degenerative abductor tears, non-surgical management is the mainstay of treatment. Nonoperative treatment involves physical therapy, cortisone injections, local anesthetic injections, and nonsteroidal antiinflammatory medications $[12,25]$. Non-surgical management has been largely successful in patients with GTPS; trochanteric bursitis touts a similar response [9,26]. Adjuncts like plateletrich plasma injections continue to be investigated for many musculoskeletal diseases including tendinopathies but have not shown promise $[12,27,28]$.

Failure of non-surgical management of GTPS should raise suspicion of abductor tendinopathy and lower the threshold for obtaining advanced imaging. Patients who fail conservative management with degenerative tears diagnosed on MRI may be candidates for surgical repair. Early surgical repair should be considered before the onset of fatty muscular atrophy [16]. Open surgical repair has been the classic technique for treating abductor tendon tears since Kagan et al. $[4,17]$ published the first case series of open repairs for GTPS in 1999. Historically, the open technique has championed a high level of patient satisfaction with objective merits such as quantifiable improvements in abductor tendon strength and gait [12,2931]. In 2009, Voos et al. [4,32] published the first case series that detailed treatment of hip abductor tears arthroscopically. Since that time, few studies have objectively compared the two techniques in the treatment of GTPS.

Two systematic reviews published in 2015 in Arthroscopy: The Journal of Arthroscopic and Related Surgery by Alpaugh et al. and Chandrasekaran et al. [1,4] are among the first to discern between outcomes of open and arthroscopic surgical repair of the hip abductors. Alpaugh et al. [4] database search and inclusion criteria yielded 8 articles. A total of 174 hips were reviewed; 135 underwent open repairs and 39 received arthroscopic repairs [4]. No significant differences were noted between the two techniques in postoperative outcomes such as the modified Harris Hip Score, Oxford Hip Score, Hip Outcome Score, and pain reduction after open and endoscopic reapir. 4 However, complication rates were found to be higher in the open repair group where the rate of tendon retear after open surgical repair was $9 \%$ (12 of 135 hips) versus no reported tendon retears following arthroscopic repair. 4

Chandrasekaran et al. [1] conducted a review of 7 of the 8 articles examined by Alpaugh et al. and arrived at similar conclusions of no significant differences between open and closed techniques as measured functionally by measures such as the Merle d'Aubigne-Postel score, Hip Outcome Score, modified Harris Hip Score, and visual analog pain scale. Chandrasekaran et al. [1] did identify a significant retear rate in patients receiving open repair where 10 out of 128 patients versus 0 reported retears in endoscopic cases. As well, 4 of the 128 open cases were complicated by infection or hematoma compared to 0 such complications in the endoscopic group [1]. While these findings and sample sizes merit further exploration, this data indicates a benefit afforded by arthroscopic repair that should impact selection of surgical technique in treating GTPS.

\section{Our Approach}

The vast majority of patients with GTPS can be treated conservatively by the methods described. Our general approach involves steroid injection and physical therapy. We will repeat the injection if necessary, but those patients that require early repeat injections often will have significant tears in the abductors. For those patients we will obtain an MRI. We also will obtain an early MRI for those patients with a positive Trendelenburg sign and gait. If the MRI demonstrates partial tears or mild degeneration, we will continue with nonoperative treatment. For those that do not respond to nonoperative management or demonstrate a full thickness tear, we proceed with arthroscopic intervention 


\section{Orthopedics and Rheumatology Open Access Journal}

provided there is no significant fatty atrophy within the muscle. The results of arthroscopic treatment have been good, but there is a significant learning curve compared to the open procedure.

\section{Conclusion}

Lateral sided hip pain is a common orthopaedic complaint that should be specifically diagnosed through history of present illness, physical exam, and potentially imaging. GTPS occurs with an incidence of 1.8 per 1000 people and encompasses several diseases including trochanteric bursitis, abductor tears, and hip snapping [7-11]. Common intraarticular pathologies such as labral tears, femoroacetabular impingement, and osteoarthritis must be excluded. Abductor tears represent a common cause of GTPS that can be accurately diagnosed on physical exam with a positive trendelenburg's sign, which is $72.7 \%$ sensitive and $76.9 \%$ to detecting gluteus medius tears [5-12]. Peritrochanteric injections that relieve pain but not weakness of the abductors may also indicate abductor insufficiency [22]. The mainstay of treatment for GTPS is non-operative which includes antiinflammatories, physical therapy, and injections [12]. Failure of conservative therapy may warrant further investigation including imaging. MRI findings of T2 hyperintensity superior to the greater trochanter are $73 \%$ sensitive and $95 \%$ specific to tears of the gluteus medius and minimus tendons [6]. Once a diagnosis of abductor tear is made, surgical treatment can include open and arthroscopic repair.

\section{References}

1. Chandrasekaran S, Lodhia P, Gui C, Vemula SP, Martin TJ, et al. (2015) Outcomes of open versus endoscopic repair of abductor muscle tears of the hip: A systematic review. Arthroscopy 31(10): 2057-2067.

2. Bunker TD (1997) Frozen shoulder: Unravelling the enigma. Ann R Coll Surg Engl 79(3): 210-213.

3. Farr D, Selesnick H, Janecki C, Cordas D (2007) Arthroscopic bursectomy with concomitant iliotibial band release for the treatment of recalcitrant trochanteric bursitis. Arthroscopy 23(8): 905.e1-5.

4. Alpaugh K, Chilelli BJ, Xu S, Martin SD (2015) Outcomes after primary open or endoscopic abductor tendon repair in the hip: A systematic review of the literature. Arthroscopy 31(3): 530-540.

5. Bird PA, Oakley SP, Shnier R, Kirkham BW (2001) Prospective evaluation of magnetic resonance imaging and physical examination findings in patients with greater trochanteric pain syndrome. Arthritis Rheum 44(9): 2138-2145.

6. Cvitanic O, Henzie G, Skezas N, Lyons J, Minter J (2004) MRI diagnosis of tears of the hip abductor tendons (gluteus medius and gluteus minimus). AJR Am J Roentgenol 182(1): 137-143.

7. Williams BS, Cohen SP (2009) Greater trochanteric pain syndrome: A review of anatomy, diagnosis and treatment. Anesth Analg 108(5): 1662-1670.

8. Frontera WR, Silver JK, Rizzo TD (2008) Essentials of physical medicine and rehabilitation musculoskeletal disorders, pain, and rehabilitation. Philadelphia, USA.

9. Redmond JM, Chen AW, Domb BG (2016) Greater trochanteric pain syndrome. J Am Acad Orthop Surg 24(4): 231-240.

10. Strauss EJ, Nho SJ, Kelly BT (2010) Greater trochanteric pain syndrome. Sports Med Arthrosc 18(2): 113-119.
11. Domb BG, Nasser RM, Botser IB (2010) Partial-thickness tears of the gluteus medius: Rationale and technique for trans-tendinous endoscopic repair. Arthroscopy 26(12): 1697-1705.

12. Chandrasekaran S, Gui C, Hutchinson MR, Lodhia P, Suarez-Ahedo C, et al. (2015) Outcomes of endoscopic gluteus medius repair: Study of thirty-four patients with minimum two-year follow-up. J Bone Joint Surg Am 97(16): 1340-1347.

13. Lequesne M, Mathieu P, Vuillemin-Bodaghi V, Bard H, Djian P (2008) Gluteal tendinopathy in refractory greater trochanter pain syndrome: Diagnostic value of two clinical tests. Arthritis Rheum 59(2): 241-246.

14. Leonard MH (1958) Trochanteric syndrome; calcareous and noncalcareous tendonitis and bursitis about the trochanter major. J Am Med Assoc 168(2): 175-177.

15. Silva F, Adams T, Feinstein J, Arroyo RA (2008) Trochanteric bursitis: Refuting the myth of inflammation. J Clin Rheumatol 14(2): 82-86.

16. Lachiewicz PF (2011) Abductor tendon tears of the hip: Evaluation and management. J Am Acad Orthop Surg 19(7): 385-391.

17. Kagan A (1999) Rotator cuff tears of the hip. Clin Orthop Relat Res (368): 135-140.

18. Bunker TD, Esler CN, Leach WJ (1997) Rotator-cuff tear of the hip. J Bone Joint Surg Br 79(4): 618-620.

19. Mulliken BD, Rorabeck CH, Bourne RB, Nayak N (1998) A modified direct lateral approach in total hip arthroplasty: A comprehensive review. J Arthroplasty 13(7): 737-747.

20. Hardinge K (1982) The direct lateral approach to the hip. J Bone Joint Surg Br 64(1): 17-19.

21. Tibor LM, Sekiya JK (2008) Differential diagnosis of pain around the hip joint. Arthroscopy 24(12): 1407-1421.

22. El-Husseiny M, Patel S, Rayan F, Haddad F (2011) Gluteus medius tears: An under-diagnosed pathology. Br J Hosp Med (Lond) 72(1): 12-16.

23. Sutter R, Kalberer F, Binkert CA, Graf N, Pfirrmann CW, et al. (2013) Abductor tendon tears are associated with hypertrophy of the tensor fasciae latae muscle. Skeletal Radiol 42(5): 627-633.

24. Long SS, Surrey DE, Nazarian LN (2013) Sonography of greater trochanteric pain syndrome and the rarity of primary bursitis. AJR Am J Roentgenol 201(5): 1083-1086.

25. Margo K, Drezner J, Motzkin D (2003) Evaluation and management of hip pain: An algorithmic approach. J Fam Pract 52(8): 607-617.

26. Lustenberger DP, Ng VY, Best TM, Ellis TJ (2011) Efficacy of treatment of trochanteric bursitis: A systematic review. Clin J Sport Med 21(5): 447-453.

27. Maffulli N, Longo UG, Denaro V (2010) Novel approaches for the management of tendinopathy. J Bone Joint Surg Am 92(15): 26042613.

28. de Vos RJ, Weir A, van Schie HT, Bierma-Zeinstra SM, Verhaar JA, et al. (2010) Platelet-rich plasma injection for chronic achilles tendinopathy: A randomized controlled trial. JAMA 303(2): 144-149.

29. Davies H, Zhaeentan S, Tavakkolizadeh A, Janes G (2009) Surgical repair of chronic tears of the hip abductor mechanism. Hip Int 19(4): 372-376.

30. Davies JF, Stiehl JB, Davies JA, Geiger PB (2013) Surgical treatment of hip abductor tendon tears. J Bone Joint Surg Am 95(15): 1420-1425.

31. Walsh MJ, Walton JR, Walsh NA (2011) Surgical repair of the gluteal tendons: A report of 72 cases. J Arthroplasty 26(8): 1514-1519.

32. Voos JE, Shindle MK, Pruett A, Asnis PD, Kelly BT (2009) Endoscopic repair of gluteus medius tendon tears of the hip. Am J Sports Med 37(4): 743-747. 